\title{
RECURRENCE FOR STATIONARY GROUP ACTIONS
}

\author{
HILLEL FURSTENBERG AND ELI GLASNER \\ In memory of Leon Ehrenpreis.
}

\begin{abstract}
Using a structure theorem from $[3$ we prove a version of multiple recurrence for sets of positive measure in a general stationary dynamical system.
\end{abstract}

\section{Contents}

Introduction

1. Poincaré recurrence for stationary actions 3

2. Multiple recurrence for SAT actions 4

3. A Structure theorem for Stationary Actions 6

Factors and the disintegration of measures 6

Topological models

The structure theorem

4. Multiple Recurrence for Stationary Actions 8

References 10

\section{INTRODUCTION}

The celebrated theorem of E. Szemerédi regarding the existence of long arithmetical progressions in subsets of the integers having positive (upper) density is known to be equivalent to a statement involving "multiple recurrence" in the framework of dynamical systems theory (see e.g. [2]). Stated precisely this is the following assertion:

Theorem 0.1. Let $(X, \mathcal{B}, \mu, T)$ be a measure preserving dynamical system; i.e., $(X, \mathcal{B}, \mu)$ is a probability space and $T: X \rightarrow X$ a measure preserving mapping of $X$ to itself. If $A \in \mathcal{B}$ is a measurable subset

Date: September 21, 2011.

2000 Mathematics Subject Classification. Primary 22D05, 37A30, 37A50. Secondary 22D40, 37A40.

Key words and phrases. Stationary dynamical systems, Szemerédi theorem, SAT, multiple recurrence. 
with $\mu(A)>0$, then for any $k=1,2,3, \ldots$ there exists $m \in \mathbb{N}=$ $\{1,2,3, \ldots\}$ with

$$
\mu\left(A \cap T^{-m} A \cap T^{-2 m} A \cap \cdots \cap T^{-k m}\right)>0 .
$$

The case $k=1$ is the "Poincaré recurrence theorem" and is an easy exercise in measure theory. The general case is more recondite (see e.g. [2]). In principle recurrence phenomena make sense in the framework of more general group actions and we can inquire what is the largest domain of their validity. Specifically if a group $G$ acts on a measure space $(X, \nu)$ (we have suppressed the $\sigma$-algebra of measurable sets) with $(g, x) \rightarrow T_{g} x$ by non-singular maps $\left\{T_{g}\right\}$, and $A$ is a measurable subset of $X$ with $\nu(A)>0$, under what conditions can we find for large $k$ an element $g \in G, g \neq$ identity, with

$$
\nu\left(A \cap T_{g}^{-1} A \cap T_{g}^{-2} A \cap \cdots \cap T_{g}^{-k} A\right)>0 ?
$$

Some conditions along the line of measure preservation will be necessary. Without this we could take $G=\mathbb{Z}, X=\mathbb{Z} \cup\{\infty\}, \quad \forall t, n \in$ $\mathbb{Z}, T_{t} n=n+t, n \neq \infty, T_{t} \infty=\infty$, and $\nu(\{n\})=\frac{1}{3 \cdot 2^{|n|}}, \nu(\{\infty\})=0$. Here no $t \neq 0$ will satisfy $T_{t}(\{n\}) \cap\{n\} \neq 0$.

The present work extends an earlier paper on "stationary" systems (3]). Here we shall show that quite generally, under the hypothesis of "stationarity", which we shall presently define, one obtains a version of multiple recurrence for sets of positive measure.

We recall the basic definitions here, although we will rely on the treatment in [3] for fundamental results. Throughout $G$ will represent a locally compact, second countable group, and $\mu$ a fixed probability measure on Borel sets of $G$. We consider measure spaces $(X, \nu)$ on which $G$ acts measurably, i.e. the map $G \times X \rightarrow X$ which we denote $(g, x) \rightarrow g x$ is measurable, and so the convolution of the measure $\mu$ on $G$ and $\nu \in \mathcal{P}(X), \mu * \nu$, is defined as the image of $\mu \times \nu$ on $X$ under this map; thus $\mu * \nu$ is again a probability measure on $X$, an element of $\mathcal{P}(X)$. We will always assume that $G$ acts on $(X, \nu)$ by non-singular transformations; i.e., $\nu(A)=0$ implies $\nu(g A)=0$ for a measurable $A \subset X$ and $g \in G$.

Definition 0.2. When $\mu * \nu=\nu$ we say that $(X, \nu)$ is a stationary $(G, \mu)$ space.

This can be interpreted as saying that $\nu$ is invariant "on the average". It is also equivalent to the statement that for measurable $A \subset X$

$$
\nu(A)=\int_{G} \nu\left(g^{-1} A\right) d \mu(g) .
$$


Associated with the space $(G, \mu)$ we will consider the probability space

$$
(\Omega, P)=(G, \mu) \times(G, \mu) \times \cdots
$$

where we will denote the random variables representing the coordinates of a point $\omega \in \Omega$ by $\left\{\xi_{1}(\omega), \xi_{2}(\omega), \ldots, \xi_{n}(\omega), \ldots\right\}$. We will draw heavily on the "martingale convergence theorem" which for our purposes can be formulated:

Theorem 0.3 (Martingale convergence theorem (MCT)). Let $\left\{F_{n}(\omega)\right\}_{n \in \mathbb{N}}$ be a sequence of uniformly bounded, measurable, real valued functions on $\Omega$ with $F_{n}$ measurable with respect to $\xi_{1}, \xi_{2}, \ldots, \xi_{n}$ and such that

$$
F_{n}\left(\xi_{1}, \xi_{2}, \ldots, \xi_{n}\right)=\int_{G} F_{n+1}\left(\xi_{1}, \xi_{2}, \ldots, \xi_{n}, \eta\right) d \mu(\eta) .
$$

(Such a sequence is called a martingale.) Then with probability one, the sequence $\left\{F_{n}(\omega)\right\}$ converges almost surely to a limit $F(\omega)$ satisfying:

$$
\mathbb{E}(F)=\int F(\omega) d P(\omega)=\int_{G} F_{1}(\eta) d \mu(\eta) .
$$

The theory of stationary actions is intimately related to boundary theory for topological groups and the theory of harmonic functions. For details we refer the reader to [1].

\section{Poincaré ReCURRenCe For Stationary aCtions}

A first application will be a proof of a particular version of the Poincaré recurrence phenomenon for stationary actions.

Theorem 1.1. Let $G$ be an infinite discrete group and let $\mu$ be a probability measure on $G$ whose support $S(\mu)$ generates $G$ as a group. Let $(X, \nu)$ be a stationary space for $(G, \mu)$ and let $A \subset X$ be a measurable subset with $\nu(A)>0$. Then there exists $g \in G, g \neq i$ identity, with $\nu\left(A \cap g^{-1} A\right)>0$.

We start with a lemma.

Lemma 1.2. If $\Sigma(\mu)$ is the semigroup in $G$ generated by $S(\mu)$, there exists a sequence of elements $\alpha_{1}, \alpha_{2}, \alpha_{3}, \cdots \in \Sigma(\mu)$ such that no product $\alpha_{i_{1}} \alpha_{i_{2}} \cdots \alpha_{i_{n}}$ with $i_{1}<i_{2}<i_{3}<\cdots<i_{n}$ equals the identity element of $G$.

Proof. The semigroup $\Sigma(\mu)$ is infinite since a finite subsemigroup of a group is a group. We proceed inductively so that having defined $\alpha_{1}, \alpha_{2}, \ldots, \alpha_{n}$ where products don't degenerate, we can find $\alpha_{n+1} \in$ $\Sigma(\mu)$ so that no product

$$
\alpha_{i_{1}} \alpha_{i_{2}} \cdots \alpha_{i_{s}} \alpha_{n+1}=\mathrm{id}
$$


there being only finitely many values to avoid.

Proof of the theorem 1.1: The proof is based on two ingredients. First, if we define functions on $\Omega$ by

$$
F_{n}(\omega)=\nu\left(\xi_{n}^{-1} \xi_{n-1}^{-1} \cdots \xi_{1}^{-1} A\right)
$$

then by (0.1), the sequence $\left\{F_{n}\right\}$ forms a martingale. The second ingredient is the fact that in almost every sequence $\xi_{1}(\omega), \xi_{2}(\omega), \ldots$, $\xi_{n}(\omega), \ldots$ every word in the "letters" of $S(\mu)$ appears infinitely far out, and then every element in $\Sigma(\mu)$ appears as a partial product. Now let $f(\omega)=\lim F_{n}(\omega)$, which by the MCT is defined almost everywhere, then $\mathbb{E}(f)=\int \nu\left(g^{-1} A\right) d \mu(g)=\nu(A)>0$. So, if $\delta=\nu(A) / 2$, there will be a random variable $n(\omega)$ which is finite with positive probability so that for $n>n(\omega)$

$$
\nu\left(\xi_{n}^{-1} \xi_{n-1}^{-1} \cdots \xi_{1}^{-1} A\right)>\delta .
$$

Now choose $\alpha_{1}, \alpha_{2}, \alpha_{3}, \cdots \in \Sigma(\mu)$ as in the foregoing lemma, and let $N>1 / \delta$. With positive probability there is $l \geq n(\omega)$ and $0=r_{0}<$ $r_{1}<r_{2}<\cdots<r_{N}$ so that in $\Sigma(\mu)$,

$$
\xi_{l+r_{i-1}+1}(\omega) \xi_{l+r_{i-1}+2}(\omega) \cdots \xi_{l+r_{i}}(\omega)=\alpha_{i},
$$

for $i=1,2, \ldots, N-1$. By definition of $n(\omega)$

$$
\nu\left(\alpha_{i}^{-1} \cdots \alpha_{l}^{-1} \beta^{-1} A\right)>\delta \quad i=1,2, \ldots, N,
$$

where $\beta=\xi_{1} \xi_{2} \cdots \xi_{l-1}$. But this yields $N$ sets of measure $>1 / N$ in $X$ and we conclude that for some $i<j$

$$
\nu\left(\alpha_{i}^{-1} \cdots \alpha_{l}^{-1} \beta^{-1} A \cap \alpha_{j}^{-1} \cdots \alpha_{l}^{-1} \beta^{-1} A\right)>0 .
$$

This however implies that for a conjugate $\gamma$ of the product $\alpha_{j}^{-1} \alpha_{j-1}^{-1} \cdots \alpha_{i+1}^{-1}$ we have $\nu(A \cap \gamma A)>0$. Here $\gamma \neq$ id since by construction $\alpha_{i+1} \alpha_{i+2} \cdots \alpha_{j}$ $\neq \mathrm{id}$.

\section{Multiple ReCURREnCE FOR SAT ACtions}

Our main result is a multiple recurrence theorem for stationary actions. We proceed step by step proving the theorem first for the special category of actions known as SAT actions. These were introduced by Jaworski in 4].

Definition 2.1. The action of a group $G$ on a probability measure space $(X, \nu)$ is SAT (strongly approximately transitive) if for every measurable $A \subset X$ with $\nu(A)>0$, we can find a sequence $\left\{g_{n}\right\} \subset G$ with $\nu\left(g_{n} A\right) \rightarrow 1$.

We now have a second recurrence result: 
Theorem 2.2. If $(X, \nu)$ is a probability measure space on which the group $G$ acts by non-singular transformations and the $G$ action is $S A T$, then for every measurable $A \subset X$ with $\nu(A)>0$ and any integer $k \geq 1$, there is a $\gamma \in G, \gamma \neq \mathrm{id}$ with

$$
\nu\left(A \cap \gamma^{-1} A \cap \gamma^{-2} A \cap \cdots \cap \gamma^{-k} A\right)>0 .
$$

Moreover if $F$ is any finite subset of $G, \gamma$ can be chosen outside of F.

We use the following basic lemma from measure theory.

Lemma 2.3. If $\sigma: X \rightarrow X$ is a non-singular transformation with respect to a measure $\nu$ on $X$, then for any $\epsilon>0$, there exists a $\delta>0$ so that $\nu(A)<\delta$ implies $\nu(\sigma A)<\epsilon$.

Proof. If such a $\delta$ did not exist we could find $B \subset X$ with $\nu(B)=0$ and $\nu(\sigma B) \geq \epsilon$.

Proposition 2.4. Assume $G$ acts on $(X, \nu)$ by non-singular transformations and let $\gamma_{1}, \gamma_{2}, \ldots, \gamma_{k} \in G$. There exists $\delta>0$ so that if $\nu(B)>1-\delta$ then

$$
\nu\left(\gamma_{1} B \cap \gamma_{2} B \cap \cdots \cap \gamma_{k} B\right)>0 .
$$

Proof. The desired inequality will take place provided the measure of each $\gamma_{i} B^{\prime}$ is less than $1 / k$, where $B^{\prime}=X \backslash B$. By Lemma 2.3 this will hold if $\nu\left(B^{\prime}\right)$ is sufficiently small.

Proof of the theorem 2.2: Let $\sigma \neq$ id be any element of $G$. Apply Proposition 2.4 with $\gamma_{0}=\mathrm{id}, \gamma_{i}=\sigma^{-i}, i=1,2, \ldots, k$ and find $\delta>0$ so that $\nu(B)>1-\delta$ implies

$$
\nu\left(B \cap \sigma B \cap \sigma^{2} B \cap \cdots \cap \sigma^{k} B\right)>0 .
$$

Use the SAT property to find $g \in G$ with $\nu\left(g^{-1} A\right)>1-\delta$. Then

$$
\nu\left(g^{-1} A \cap \sigma g^{-1} A \cap \sigma^{2} g^{-1} A \cap \cdots \cap \sigma^{k} g^{-1} A\right)>0 .
$$

Applying $g$ to the set appearing here we get:

$$
\nu\left(A \cap g \sigma g^{-1} A \cap g \sigma^{2} g^{-1} A \cap \cdots \cap g \sigma^{k} g^{-1} A\right)>0 .
$$

Letting $\gamma=g \sigma^{-1} g^{-1}$ we obtain the desired result.

We turn now to the last statement of the theorem. One sees easily that if $G$ has a non-trivial SAT action then $G$ is infinite. Let $H$ be a finite subset of $G$ with greater cardinality than $F$. Now in the foregoing discussion we consider a sequence $\left\{g_{n}\right\}$ in $G$ with $\nu\left(g_{n}^{-1} A\right) \rightarrow 1$; then for any $\sigma$, if $n$ is sufficiently large if we take $\gamma=g_{n} \sigma g_{n}^{-1}$ for large $n$ we will get (2.1). We claim that $\sigma$ can be chosen so that for an infinite subsequence $\left\{n_{j}\right\}$ we will have $g_{n_{j}} \sigma g_{n_{j}}^{-1} \notin F$. For this we simply 
consider the sets $\left\{g_{n} H g_{n}^{-1}\right\}$ each of which has some element outside of $F$. $\left\{n_{j}\right\}$ is then a sequence for which there is a fixed $\sigma \in H$ with $g_{n_{j}} \sigma g_{n_{j}}^{-1} \notin F$. This completes the proof.

\section{A Structure theorem for Stationary Actions}

In order to formulate our structure theorem we will introduce a few definitions and some well known basic tools from the general theory of dynamical systems.

\section{Factors and the disintegration of measures.}

Definition 3.1. Let $(X, \nu)$ and $(Y, \rho)$ be two $(G, \mu)$ spaces. A measurable map $\pi:(X, \nu) \rightarrow(Y, \rho)$ is called a factor map, or an extension, depending on the view point, if it intertwines the group actions: for every $g \in G, g \pi(x)=\pi(g x)$ for $\nu$ almost every $x \in X$.

Definition 3.2. If $(Y, \rho)$ is a factor of $(X, \nu)$ we can decompose the measure $\nu$ as $\nu=\int_{Y} \nu_{y} d \rho(y)$, where the $\nu_{y}$ are probability measures on $X$ with $\nu_{y}\left(\pi^{-1}(y)\right)=1$ and the map $y \mapsto \nu_{y}$ is measurable from $Y$ into the space of probability measures on $X$, equipped with its natural Borel structure. We say $(X, \nu)$ is a measure preserving extension of $(Y, \rho)$ if for each $g \in G, g \nu_{y}=\nu_{g y}$ for almost every $y \in Y$. Note that a stationary system $(X, \nu)$ is measure preserving (i.e. $g \nu=\nu$ for every $g \in G)$ if and only if the extension $\pi: X \rightarrow Y$, where the factor $(Y, \rho)$ is the trivial one point system, is a measure preserving extension.

Topological models. We begin this subsection with some remarks regarding stationary actions of $(G, \mu)$ on $(X, \nu)$ in the case that $X$ is a compact metric space. We then speak of a topological stationary system. In this case we can form the measure-valued martingale

$$
\theta_{n}(\omega)=\xi_{1} \xi_{2} \cdots \xi_{n} \nu
$$

The martingale convergence theorem is valid also in this context by the separability of $\mathrm{C}(X)$, and so we obtain a measure-valued random variable $\theta(\omega)=\lim _{n \rightarrow \infty} \theta_{n}(\omega)$.

Definition 3.3. A topological stationary system $(X, \nu)$ is proximal if with probability 1 , the measure $\theta(\omega)$ is a Dirac measure: $\theta(\omega)=\delta_{z(\omega)}$.

Definition 3.4. A stationary system $(X, \nu)$ is proximal if every compact metric factor $\left(X^{\prime}, \nu^{\prime}\right)$ is proximal.

Definition 3.5. Let $(X, \nu)$ and $\left(X^{\prime}, \nu^{\prime}\right)$ be two $(G, \mu)$ stationary systems, and suppose that $X^{\prime}$ is a compact metric space. We say that 
the stationary system $\left(X^{\prime}, \nu^{\prime}\right)$ is a topological model for $(X, \nu)$ if there is an isomorphism of the measure spaces $\phi:(X, \nu) \rightarrow\left(X^{\prime}, \nu^{\prime}\right)$ which intertwines the $G$ actions.

The following proposition is well known and has several proofs. We will be content here with just a sketch of an abstract construction.

Proposition 3.6. Every $(G, \mu)$ system $(X, \nu)$ admits a topological model. Moreover, if $A \subset X$ is measurable we can find a topological model $\phi:(X, \nu) \rightarrow\left(X^{\prime}, \nu^{\prime}\right)$ such that the set $A^{\prime}=\phi(A)$ is a clopen subset of the compact space $X^{\prime}$.

Proof. Choose a sequence of functions $\left\{f_{n}\right\} \subset L_{\infty}(X, \nu)$ which spans $L_{2}(X, \nu)$, with $f_{1}=\mathbf{1}_{A}$. Let $G_{0} \subset G$ be a countable dense subgroup and let $\mathcal{A}$ be the $G_{0}$-invariant closed unital $C^{*}$-subalgebra of $L_{\infty}(X, \nu)$ which is generated by $\left\{f_{n}\right\}$. We let $X^{\prime}$ be the, compact metric, Gelfand space which corresponds to the $G$-invariant, separable, $C^{*}$-algebra $\mathcal{A}$. Since $f_{1}^{2}=f_{1}$ we also have $\tilde{f}_{1}^{2}=\tilde{f}_{1}$, where the latter is the element of $C\left(X^{\prime}\right)$ which corresponds to $f_{1}$. Since $\tilde{f}_{1}$ is continuous it follows that $A^{\prime}:=\left\{x^{\prime}: \tilde{f}_{1}\left(x^{\prime}\right)=1\right\}$ is indeed a clopen subset of $X^{\prime}$ with $\tilde{f}_{1}=\mathbf{1}_{A^{\prime}}$. The probability measure $\nu^{\prime}$ is the measure which corresponds, via Riesz' theorem, to the linear functional $\tilde{f} \mapsto \int f d \nu$.

Proposition 3.7. If $(X, \nu)$ is a proximal stationary system for $(G, \mu)$ then the action of $G$ on $(X, \nu)$ is $S A T$.

Proof. Let $A$ be a measurable subset of $X$ with $\nu(A)>0$. There is a topological model $\left(X^{\prime}, \nu^{\prime}\right)$ of $(X, \nu)$ such that $A$ is the pullback of a closed-open set $A^{\prime}$ with $\nu^{\prime}\left(A^{\prime}\right)=\nu(A)$. As in section 1 we form the martingale $\nu^{\prime}\left(\xi_{n}^{-1} \xi_{n-1}^{-1} \cdots \xi_{1}^{-1} A^{\prime}\right)$ which converges to $\theta(\omega)(A)=\delta_{z^{\prime}(\omega)}\left(A^{\prime}\right)$, since by the proximality of $(X, \nu)$, the topological factor $\left(X^{\prime}, \nu^{\prime}\right)$ is proximal. Now the latter limit is 0 or 1 and since the expectation of $\nu^{\prime}\left(\xi_{n}^{-1} \xi_{n-1}^{-1} \cdots \xi_{1}^{-1} A\right)$ is $\nu(A)>0$, there is positive probability that $z^{\prime}(\omega) \in A^{\prime}$. When this happens

$$
\nu\left(\xi_{n}^{-1} \xi_{n-1}^{-1} \cdots \xi_{1}^{-1} A\right)=\nu^{\prime}\left(\xi_{n}^{-1} \xi_{n-1}^{-1} \cdots \xi_{1}^{-1} A^{\prime}\right) \rightarrow 1 .
$$

This proves that the action is SAT.

The structure theorem. We now reformulate the structure theorem (theorem 4.3) of [3] to suit our needs. (The theorem in [3] gives more precise information.)

Theorem 3.8. Every stationary system is a factor of a stationary system which is a measure preserving extension of a proximal system.

Alternatively, in view of proposition 3.7 . 
Theorem 3.9. If $(X, \nu)$ is a stationary action of $(G, \mu)$, there is an extension $\left(X^{*}, \nu^{*}\right)$ of $(X, \nu)$ which is a measure preserving extension of an $S A T$ action of $G$ on a stationary space $(Y, \rho)$.

This is the basic structure theorem which we will use to deduce a general multiple recurrence result for stationary actions.

\section{Multiple Recurrence for Stationary Actions}

We recall the terminology of [3]:

Definition 4.1. A $(G, \mu)$ stationary action of on $(X, \nu)$ is standard if $(X, \nu)$ is a measure preserving extension of a proximal action.

Since proximality implies SAT we can extend this notion and replace "proximal" by SAT. Theorem 3.8 asserts that every stationary action has a standard extension. The nature of recurrence phenomena is such that if such a phenomenon is valid for an extension of a system, it is valid for the system. Precisely, if $\pi: X \rightarrow X^{\prime}$ and $A^{\prime} \subset X^{\prime}$ and for the pullback $A=\pi^{-1}\left(A^{\prime}\right)$ and a set $g_{1}, g_{2}, \ldots, g_{k}$, we have $\nu\left(g_{1}^{-1} A \cap\right.$ $\left.g_{2}^{-1} A \cap \cdots \cap g_{k}^{-1} A\right)>0$, then $\nu^{\prime}\left(g_{1}^{-1} A^{\prime} \cap g_{2}^{-1} A^{\prime} \cap \cdots \cap g_{2}^{-1} A^{\prime}\right)>0$. It follows now from theorem 3.8 that for a general multiple recurrence theorem for stationary actions, it will suffice to treat standard actions. Using the definition of a standard action we will take advantage of the multiple recurrence theorem proved in Section 2 for SAT actions and show that this now extends to any standard action. For this we use a lemma which is based on Szemerédi's theorem. By the latter there is a function $N(\delta, \ell)$, for $\delta>0$ and $\ell$ a natural number, so that for $n \geq N(\delta, \ell)$, if $E \subset\{1,2,3, \ldots, n\}$ with $|E| \geq \delta n$ then $E$ contains an $\ell$-term arithmetic progression. We now have

Lemma 4.2. In any probability space $(\Omega, P)$, for $n \geq N(\delta, \ell)$, if $A_{1}, A_{2}$, $\ldots, A_{n}$ are $n$ subsets of $\Omega$ with $P\left(A_{i}\right)>\delta$ for $i=1,2, \ldots, n$, then there exist $a$ and $d$ so that

$$
P\left(A_{a} \cap A_{a+d} \cap A_{a+d} \cap \cdots \cap A_{a+(\ell-1) d}\right)>0 .
$$

Proof. Set $f_{i}(x)=1_{A_{i}}(x), i=1,2, \ldots, n$, and let $E(x)=\left\{i: f_{i}(x)=\right.$ 1\}. $|E(x)|=\sum_{i=1}^{n} f_{i}(x)$ and the condition $|E(x)|>\delta n$ is implied by $F(x)=\Sigma f_{i}(x)>\delta n$. But $\int F(x) d P(x)=\Sigma P\left(A_{i}\right)>\delta n$ and so for some set $B \subset \Omega$ with $P(B)>0, F(x)>\delta n$. Thus for each $x \in B$ we have $|E(x)|>n \delta$ and there is an $\ell$-term arithmetic progression $R_{a, d}(x) \subset E(x)$, so that $x$ lies in the intersection of the $A_{r}$, as $r$ ranges 
over the arithmetic progression $R_{a, d}(x)$. There being only finitely many progressions we obtain for one of these $P\left(\bigcap_{r \in R_{a, d}} A_{r}\right)>0$.

We will need an additional hypothesis to obtain a general multiple recurrence theorem.

Definition 4.3. A group $G$ is $\mathrm{OU}$ (order unbounded) if for any integer $n$ we have for some $g \in G, g^{n} \neq i d$.

For an OU group we can find, for any given $k$, elements $\sigma \in G$ so that none of the powers $\sigma, \sigma^{2}, \ldots \sigma^{k}$ give the identity. Note that in our proof of multiple recurrence for SAT actions, theorem [2.2, we obtain, for any subset $A \subset X$ of positive measure, an element id $\neq \gamma \in G$ with:

$$
\nu\left(A \cap \gamma^{-1} A \cap \gamma^{-2} A \cap \cdots \cap \gamma^{-k} A\right)>0,
$$

where for an OU group we can demand that each $\gamma^{j} \neq i d, j=$ $1,2, \ldots, k$. In fact, in that proof we show that the element $\gamma$ can be found within the conjugacy class of any non-identity element $\sigma$ of G.

We can now prove

Theorem 4.4. Let $(X, \nu)$ represent a stationary action of $(G, \mu)$ with the elements of $G$ acting on $(X, \nu)$ by non-singular transformations and where $G$ is an $O U$ group. Let $A \subset X$ be a measurable set with $\nu(A)>0$ and let $k \geq 1$ be any integer then there exists an element $\gamma$ in $G$ with $\gamma^{j} \neq i d, j=1,2, \ldots, k$ and with

$$
\nu\left(A \cap \gamma^{-1} A \cap \gamma^{-2} A \cap \cdots \cap \gamma^{-k} A\right)>0 .
$$

Proof. We can assume $(X, \nu)$ is a measure preserving extension of $(Y, \rho)$ where the action of $G$ on $(Y, \rho)$ is SAT. Let $\pi: X \rightarrow Y$ and decompose $\nu=\int \nu_{y} d \rho(y)$. Let $A \subset X$ be given and let $\delta>0$ be such that $B=\left\{y: \nu_{y}(A)>\delta\right\}$ has positive measure. Set $N=N(\delta, k)$ in theorem 2.2 and find $\gamma$ with $\gamma^{j} \neq i d$ for $j=1,2, \ldots, N$, and with

$$
\rho\left(B \cap \gamma^{-1} B \cap \gamma^{-2} B \cap \cdots \cap \gamma^{-N} B\right)>0 .
$$

For $y \in B \cap \gamma^{-1} B \cap \cdots \cap \gamma^{-N} B$ and $j=1,2, \ldots, N$ we will have

$$
\nu_{y}\left(\gamma^{-j} A\right)=\gamma^{j} \nu_{y}(A)=\nu_{\gamma^{j} y}(A)>\delta .
$$

We now use lemma 4.2 to obtain for each $y \in B \cap \gamma^{-1} B \cap \cdots \cap \gamma^{N} B$ a $k$-term arithmetic progression $R$ of powers of $\gamma$ with $\nu_{y}\left(\bigcap_{j \in R} \gamma^{-j} A\right)>0$.

In particular $\bigcap_{j \in R} \gamma^{-j} A \neq \emptyset$ for some arithmetic progression $R=\{a, a+$ 
$d, a+2 d, \ldots, a+(k-1) d\}$ so that with $\gamma^{\prime}=\gamma^{d}$,

$$
A \cap \gamma^{\prime-1} A \cap \gamma^{\prime-2} A \cap \cdots \cap \gamma^{\prime-k} \neq \emptyset \text {. }
$$

Obtaining a non-empty intersection suffices to obtain an intersection of positive measure, and so our theorem is proved.

\section{REFERENCES}

1. H. Furstenberg, Boundary theory and stochastic processes on homogeneous spaces, Harmonic analysis on homogeneous spaces (Proc. Sympos. Pure Math., Vol. XXVI, Williams Coll., Williamstown, Mass., 1972), 193-229. Amer. Math. Soc., Providence, R.I., 1973

2. H. Furstenberg, Recurrence in ergodic theory and combinatorial number theory, Princeton university press, Princeton, N.J., 1981.

3. H. Furstenberg and E. Glasner, Stationary dynamical systems, Dynamical numbers, AMS, Contemporary Math. 532, 1-28, Providence, Rhode Island, 2010.

4. W. Jaworski Strongly approximately transitive group actions, the ChoquetDeny theorem, and polynomial growth, Pacific J. of Math., 165, (1994), 115-129.

Department of Mathematics, Hebrew University of Jerusalem, Jerusalem, ISRAEL

E-mail address: harry@math.huji.ac.il

Department of Mathematics, Tel Aviv University, Ramat Aviv, IsRAEL

E-mail address: glasner@math.tau.ac.il 